\title{
超高非线性二维材料复合光纤制造取得新进展
}

刘益春

东北师范大学物理学院, 长春 130024

\section{New Progress in the Manufacture of Ultrahigh Nonlinear Two-Dimensional Material Hybrid Fiber}

Yichun Liu

School of Physics, Northeast Normal University, Changchun 1300024, China.

Email: ycliu@nenu.edu.cn

Published online: December 17, 2020.

a
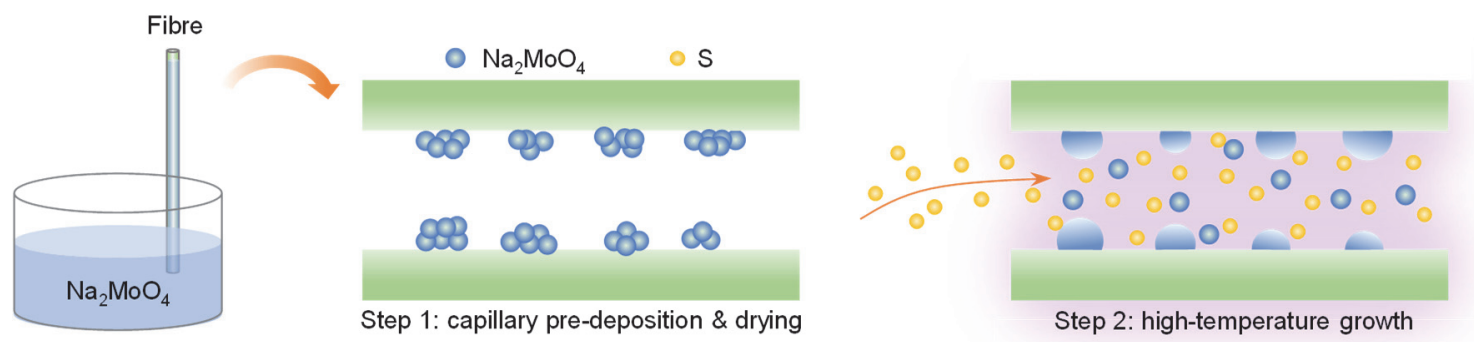

b
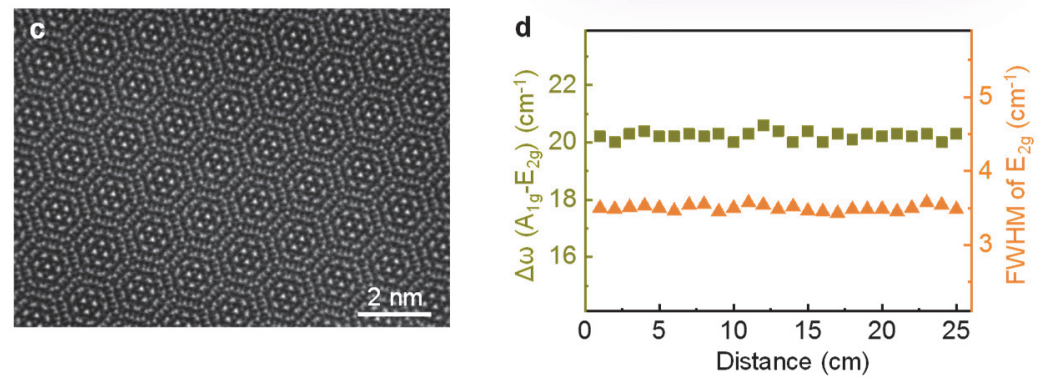

图 1 二维材料复合光纤制备。(a)两步法化学气相沉积制备 $\mathrm{MoS}_{2}$ 复合光纤示意图。(b) $50 \mu \mathrm{m}$ 孔径石英管光纤中 $\mathrm{MoS}_{2}$ 大单晶光学图像。(c) HF 刻蚀处理光纤后形成的坡塌 $\mathrm{MoS}_{2}$ 管的莫尔条纹扫描电子显微镜图像。

(d) $25 \mathrm{~cm}$ 长 $M_{0} S_{2}$ 复合光纤样品 Raman 光谱数据统计。

非线性光纤在光通讯、光传感、光学频率转 换、超快光纤激光器、光频梳及超连续激光等众多 领域展现出重要应用前景 ${ }^{1-5}$ 。然而, 目前绝大多数 光纤器件都是基于传统的石英光纤, 这些光纤仅 具有微弱的三阶非线性效应以及接近于零的二阶 非线性效应, 严重限制了它在非线性光学领域的 应用范围。目前, 提高光纤非线性效应的方法主要 分为两大类: (1)通过优化光纤结构设计, 减小光
纤的有效纤芯面积, 进而提高光纤非线性 ${ }^{6-8}$ 。(2) 通过对传统石英纤芯进行掺杂(如硫化物等)或者 直接生长非石英纤芯(如硅、锗等)来提高光纤非线 性效应 ${ }^{9-11}$ 。但这两种方法对提升光纤非线性效果 有限, 且不易规模化生产。因此, 开发新型高非线 性光纤势在必行。

近年来, 二维材料由于其优异的光电特性、超 高非线性系数和原子层厚度, 迅速掀起了与光纤 

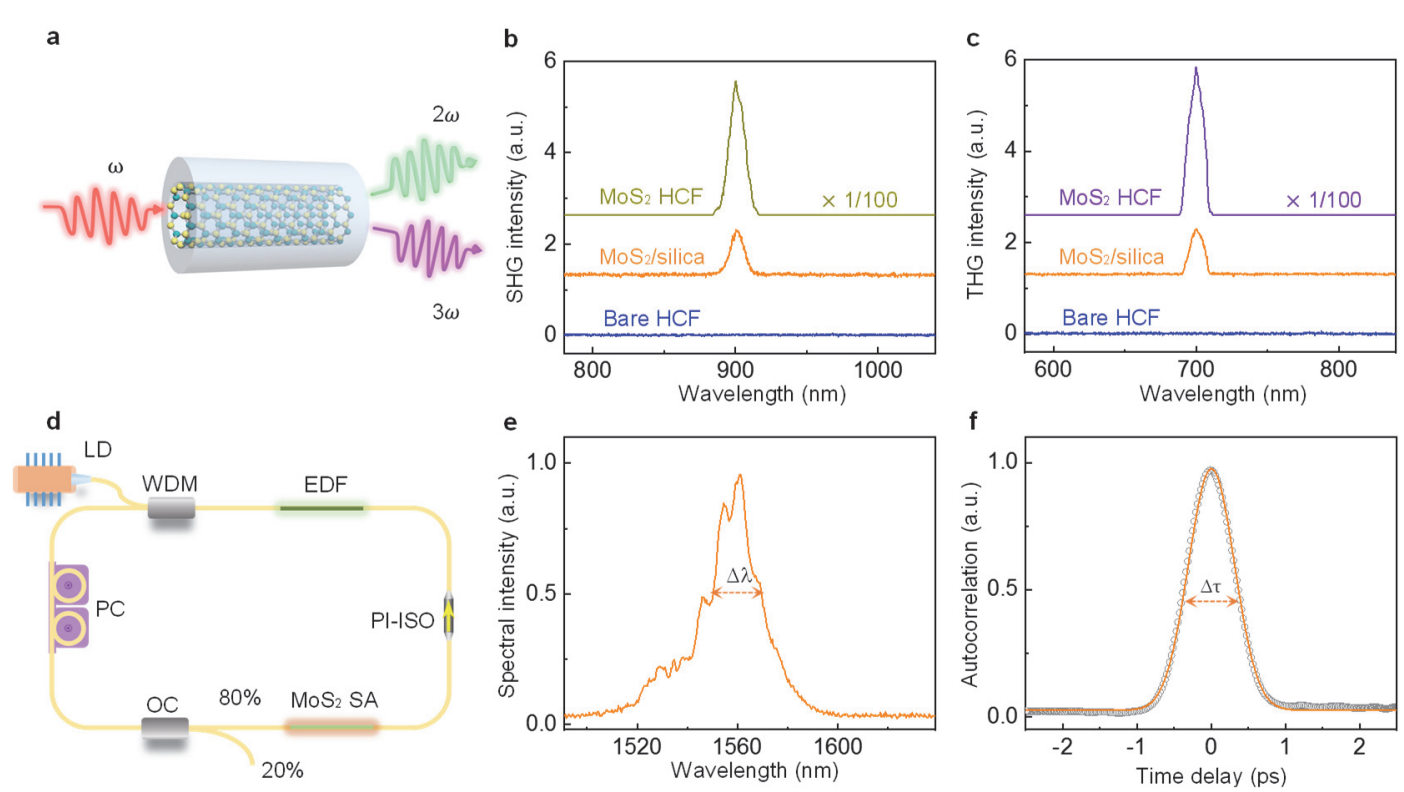

图 2 二维材料复合光纤的非线性应用。(a) $\mathrm{MoS}_{2}$ 光纤产生二次谐波和三次谐波示意图。(b-c) $\mathrm{MoS}_{2}$ 复合光纤产生的 二次谐波(b)和三次谐波(c)与平面石英祄底上 $\mathrm{MoS}_{2}$ 样品以及裸纤的比较。(d)使用 $\mathrm{MoS}_{2}$ 光纤做饱和吸收体的 全光纤锁模激光器结构示意图。(e)激光器输出光谱。(f)激光器输出脉冲的自相关曲线。

光学相结合的交叉学科领域的研究热潮。大量研 究工作通过转移或涂覆方式将二维材料和光纤结 合, 实现光纤的功能化应用, 例如四波混频和光电 调制。但这种方法一般需要改变光纤结构(例如侧 剖和拉锥光纤)来实现材料和修逝波的耦合, 往往 会影响光纤的传输能力, 产生额外的传输损耗, 且 不易高质量大规模制备。基于此, 北京大学/北京 石墨烯研究院刘忠范院士和刘开辉研究员、中国 科学院物理研究所白雪冬研究员及其合作者在 Nature Nanotechnology发表了题为 “Optical fibres with embedded two-dimensional materials for ultrahigh nonlinearity” 的文章 ${ }^{12}$, 报道了分米级超 高非线性二维材料复合光纤的制备进展, 利用该 二维材料复合光纤实现了超高非线性信号的增强 (2-3 个量级), 并且实现了真正的亚皮秒量级( 500 $\mathrm{fs}$ ) 全光纤锁模激光器, 这些应用结果展示了二维 材料复合光纤在非线性光学领域的优异性能和应 用前景。

基于北京大学/北京石墨烯研究院前期在米级 石墨烯光子晶体光纤的工作积累 ${ }^{13}$, 该研究团队利 用两步化学气相沉积法在多种类型光纤孔内壁直 接生长二维过渡金属硫族化合物(TMDs), 实现了 超高非线性二维材料复合光纤的制备(图1)。与石 墨烯光纤制备不同, TMDs光纤制备更具挑战性: 化学气相沉积生长TMDs过程, 过渡金属前驱体多 为固态(氧化钼、氧化铇等), 其饱和蒸汽压较低, 在光纤微结构中无法均匀传质。即使在低压情况
下, 也无法保证前驱体在光纤微结构中的有效传 输和均匀生长。鉴于此, 该研究团队首次提出一种 两步化学气相沉积的方法, 通过低温预涂覆过渡 金属前驱体 $\mathrm{Na}_{2} \mathrm{MoO}_{4}$, 并在高温硫化及生长, 成功 在光纤孔内壁上直接生长出单层 $\mathrm{MoS}_{2}$, 并且实现 了多种二维 TMDs材料及其合金在不同种类规格 光纤(空心石英管光纤和光子晶体光纤)中均匀全 覆盖生长, 长度最长可达 $25 \mathrm{~cm}$ 。

TMDs与光纤中传输的光通过修逝波进行耦 合, 光纤优异的光波导能力可以大大增加光与物 质的相互作用长度, 进而提高非线性效应。文章作 者分别基于非线性系数的实部和虚部进行了相应 的应用研究(图2)。(1)非线性实部: 光频转换应用 研究, 二维材料复合光纤产生了超强的二次和三 次谐波, 实验结果发现 $\mathrm{MoS}_{2}$ 复合光纤的非线性信 号比平面上 $\mathrm{MoS}_{2}$ 样品增强 300 倍且损伤阈值提高 3倍。这为未来设计新型基于光纤的非线性材料提 供了一种新的设计思路, 结合光纤结构设计的优 化, 这种新型复合光纤材料有望取代现在常用的 非线性晶体, 实现非线性光学材料领域重大突破。 (2)非线性虚部: 全光纤超快脉冲激光研究, 利用 $\mathrm{MoS}_{2}$ 复合光纤作为饱和吸收器, 实现了全光纤超 快脉冲激光输出, 其具有超短脉冲宽度 $200 \mathrm{fs}$, 高 重复频率 41 MHz, 输出功率 $6 \mathrm{~mW}$ 的优异性能。 二维材料光纤为全光纤超快脉冲激光器提供了一 种新的低成本、高性能且可重复制备的饱和吸收 器材料, 推进了全光纤锁模激光器的发展和低维 
材料关键而重要的应用。

这一工作开创性地将高非线性二维材料与光 纤复合, 不仅拓展了二维材料应用领域, 同时为光 纤器件功能化研究提供了新思路, 对新型光电子 器件相关领域具备示范和引领作用。该工作所展 示的方法不仅可以实现二维TMDs材料与光纤有 效集成及规模化制备，同时为新型非线性材料的 设计开辟了新领域, 对后续超连续光谱、高次谐波 以及基于非线性效应的新型光纤通讯提供了材料 支撑。可以预见，随着未来二维材料生长工艺的不 断提高和改进, 同时结合光纤结构设计等, 可以满 足各种类型的非线性光学和光纤光学研究需求, 推 动二维材料复合光纤在光电、非线性光学等领域 的批量制备和功能化应用。

\section{References}

(1) Cotter, D.; Manning, R.; Blow, K.; Ellis, A.; Kelly, A.; Nesset, D.; Phillips, I.; Poustie, A.; Rogers, D. Science 1999, 286, 1523. doi: $10.1126 /$ science.286.5444.1523

(2) Agrawal, G. P. J. Opt. Soc. Am. B 2011, 28, A1. doi: $10.1364 / J O S A B .28 .0000 A 1$

(3) Granzow, N.; Stark, S. P.; Schmidt, M. A.; Tverjanovich, A.; Wondraczek, L.; Russell, P. S. J. Opt. Express 2011, 19, 21003. doi: 10.1364/OE.19.021003

(4) Markos, C.; Travers, J. C.; Abdolvand, A.; Eggleton, B. J.; Bang, O.
Rev. Mod. Phys. 2017, 89, 045003.

doi: 10.1103/RevModPhys.89.045003

(5) Mollenauer, L. F. Science 2003, 302, 996. doi: $10.1126 /$ science. 1091168

(6) Skryabin, D.; Luan, F.; Knight, J.; Russell, P. S. J. Science 2003, 301, 1705. doi: 10.1126/science. 1088516

(7) Dudley, J. M.; Genty, G.; Coen, S. Rev. Mod. Phys. 2006, 78, 1135. doi: 10.1103/RevModPhys.78.1135

(8) Dudley, J. M.; Taylor, J. R. Nat. Photon. 2009, 3, 85. doi: $10.1038 /$ nphoton. 2008.285

(9) Sazio, P. J.; Amezcua-Correa, A.; Finlayson, C. E.; Hayes, J. R.; Scheidemantel, T. J.; Baril, N. F.; Jackson, B. R.; Won, D.-J.; Zhang, F.; Margine, E. R.; et al. Science 2006, 311, 1583. doi: $10.1126 /$ science. 1124281

(10) Abouraddy, A.; Bayindir, M.; Benoit, G.; Hart, S.; Kuriki, K.; Orf, N.; Shapira, O.; Sorin, F.; Temelkuran, B.; Fink, Y. Nat. Mater. 2007, 6, 336. doi: $10.1038 /$ nmat 1889

(11) Eggleton, B. J.; Luther-Davies, B.; Richardson, K. Nat. Photon. 2011, 5, 141. doi: 10.1038/nphoton.2011.309

(12) Zuo, Y.; Yu, W.; Liu, C.; Cheng, X.; Qiao, R.; Liang, J.; Zhou, X.; Wang, J.; Wu, M.; Zhao, Y.; et al. Nat. Nanotechnol. 2020, 15, 987 doi: 10.1038/s41565-020-0770-x

(13) Chen, K.; Zhou, X.; Cheng, X.; Qiao, R.; Cheng, Y.; Liu, C.; Xie, Y.; Yu, W.; Yao, F.; Sun, Z.; et al. Nat. Photon. 2019, 13, 754. doi: 10.1038/s41566-019-0492-5 